\title{
Stability Analysis of Stochastic Generalized Equation via Brouwer's Fixed Point Theorem
}

\author{
Qiang Liu, ${ }^{1,2}$ Jie Zhang $\mathbb{D}^{3}{ }^{3}$ Shuang Lin, ${ }^{4}$ and Li-wei Zhang ${ }^{1}$ \\ ${ }^{1}$ School of Mathematical Science, Dalian University of Technology, Dalian, China \\ ${ }^{2}$ School of Science, Dalian Nationalities University, Dalian, China \\ ${ }^{3}$ School of Mathematics, Liaoning Normal University, Dalian, China \\ ${ }^{4}$ School of Information Science and Engineering, Dalian Polytechnic University, Dalian, China \\ Correspondence should be addressed to Jie Zhang; zhangjie04212001@163.com
}

Received 24 October 2017; Accepted 19 February 2018; Published 26 March 2018

Academic Editor: Kishin Sadarangani

Copyright (C) 2018 Qiang Liu et al. This is an open access article distributed under the Creative Commons Attribution License, which permits unrestricted use, distribution, and reproduction in any medium, provided the original work is properly cited.

\begin{abstract}
The stochastic generalized equation provides a unifying methodology to study several important stochastic programming problems in engineering and economics. Under some metric regularity conditions, the quantitative stability analysis of solutions of a stochastic generalized equation with the variation of the probability measure is investigated via Brouwer's fixed point theorem. In particular, the error bounds described by Hausdorff distance between the solution sets are established against the variation of the probability measure. The stability results obtained are finally applied to a stochastic conic programming.
\end{abstract}

\section{Introduction}

In this paper, we focus on the following stochastic generalized equation (SGE): find $x \in \mathbb{R}^{n}$ such that

$$
0 \in \mathbb{E}_{P}[F(x, \xi(\omega))]+\mathscr{G}(x),
$$

where $F: \mathbb{R}^{n} \times \Xi \rightarrow \mathbb{R}^{m}$ is a continuous function, $\xi$ : $\Omega \rightarrow \Xi$ is a random vector defined on a probability space $(\Omega, \mathscr{F}, P)$ with support set $\Xi \subseteq \mathbb{R}^{k}, \mathbb{E}_{P}[\cdot]$ denotes the expected value with respect to $P$, and $\mathscr{G}: \mathbb{R}^{n} \rightrightarrows \mathbb{R}^{m}$ is an outer semicontinuous set-valued mapping. Throughout the paper, we assume that $\mathbb{E}_{p}[F(x, \xi(\omega))]$ is well defined for any $x \in \mathbb{R}^{n}$. To ease notation, we will use $\xi$ to denote either the random vector $\xi(\omega)$ or an element of $\mathbb{R}^{k}$ depending on the context.

Model (1) a natural extension of deterministic parametric generalized equation [1] and the study of stochastic generalized equations can be traced down to King and Rockafellar's early work [2]. In a particular case when $\mathscr{G}(\cdot)$ is a normal cone operator $\mathcal{N}_{\mathscr{K}}(\cdot)$ in which $\mathscr{K}$ is a closed convex cone in $\mathbb{R}^{m}$, (1) reduces to a stochastic variational inequality problem (SVIP) which has been intensively studied over the past few years; see for instance [3-7] and the references therein. The research ranges from numerical schemes such as stochastic approximation method and Monte Carlo method to the fundamental theory and applications.

In this paper, we concentrate our research on the stability of (1); namely, we look into the impact of variation of probability measure $P$ on the solution of the SGE. Like similar existing research in deterministic generalized equation, this kind of stability analysis would address a number of fundamental theoretical issues including robustness, accuracy, and reliability of an optimal solution or an equilibrium against errors arising from the problem data or numerical schemes. Let $Q$ denote a perturbation of the probability measure $P$. We consider the following perturbed stochastic generalized equations: find a vector $x \in \Re^{n}$ such that

$$
0 \in \mathbb{E}_{\mathrm{Q}}[F(x, \xi)]+\mathscr{G}(x) .
$$

Let $S(P)$ and $S(Q)$ denote the solution set of (1) and (2), respectively. We investigate the relationship between $S(Q)$ and $S(P)$ as $Q$ approximates $P$ under some appropriate metric.

Shapiro et al. [8] first discussed the sample average approximation (SAA) approach for (1). This method can be seen a special case of the perturbation of $P$ (see Section 4 in this paper). They carried out comprehensive analysis 
including the existence and convergence of solutions. In our previous work [9], the consistency of Lipschitz-like property of solution map to (1) and its SAA counterpart have been studied. However, the above two studies only focus on the asymptotic analysis and are not related to the quantitative stability analysis. Recently, Liu et al. [7] have studied the qualitative stability of solutions of SGE (1) with $F$ being a set-valued mapping as the underlying probability measure $P$ varies. The results are applied to study the stability of stationary points of several stochastic optimization problems.

In this paper, we follow Liu et al's approach to investigate the existence and quantitative stability analysis of solutions of problem (1) when $P$ varies under some appropriate metric. We complement the results of Liu et al. on the issues essentially on twofold: (a) we use Brouwer's fixed point theorem and metric regularity rather than Kummer's results as in [7] to derive conditions for the existence of a solution to perturbation problem (2); (b) by Brouwer's fixed point theorem, we establish the error bounds described by Hausdorff distance between solution sets, instead of the one described by distance from point to set as in [7], to show the quantitative stability of (1) when $P$ varies. We also apply the results to analyze the convergence of SAA method for a class of stochastic conic programming and establish the corresponding error bounds.

This paper is organized as follows: Section 2 gives preliminaries needed throughout the paper. In Section 3, by Brouwer's fixed point theorem, the existence of solution to perturbation problem (2) is investigated. Section 3 provides quantitative stability analysis of problem (1) when $P$ varies under some appropriate metric. In particular, error bounds described by Hausdorff distance between $S(P)$ and $S(Q)$ are established. Finally, in Section 4, we apply the results obtained to obtaining quantitative convergence analysis of SAA method for a stochastic conic programming.

\section{Preliminaries}

2.1. Notation. Throughout this paper we use the following notations. Let $\|\cdot\|$ denote the Euclidean norm of a vector or the Frobenius norm of a matrix and $d(x, D):=\inf _{x^{\prime} \in D}\left\|x-x^{\prime}\right\|$ denote the distance from point $x$ to set $D$. For a multifunction $\Phi, \operatorname{gph} \Phi$ denotes its graph and for a set $\Xi$, int $\Xi$ denotes its interior. For an extended real-valued function $\varphi: \mathbb{R}^{n} \rightarrow$ $\mathbb{R} \cup\{ \pm \infty\}, \nabla \varphi(x)$ denotes the gradient of $\varphi$ at $x$. For a continuously differentiable mapping $\phi: \mathbb{R}^{n} \rightarrow \mathbb{R}^{m}, \mathscr{J} \phi(x)$ denotes the Jacobian matrix of $\phi$ at $x$. We use $\mathscr{B}_{n}$ to denote the closed unite ball in $\mathbb{R}^{n}, \mathscr{B}(x, \delta)$ the closed ball around $x$ of radius $\delta>0$, and $I$ the identity matrix. For two sets $A, C \subset \mathbb{R}^{n}$, we denote by

$$
\mathbb{D}(A, C):=\inf \left\{t \geq 0: A \subset C+t \mathscr{B}_{n}\right\}
$$

the deviation of set $A$ from the set $C$ and

$$
\mathbb{H}(A, C):=\max \{\mathbb{D}(A, C), \mathbb{D}(C, A)\}
$$

the Hausdorff distance between $A$ and $C$. We use $A+C$ to denote the Minkowski addition of two sets; that is, $\{a+c$ : $a \in A, c \in C\}$. For the multifunction $\Phi: \mathfrak{R}^{n} \rightrightarrows \mathfrak{R}^{m}$, the set

$$
\limsup _{x \rightarrow x_{0}} \Phi(x):=\left\{y \in \mathfrak{R}^{m}: \exists x_{k} \longrightarrow x_{0}, y_{k} \longrightarrow y \text { with } y_{k} \in \Phi\left(x_{k}\right), \forall k\right\}
$$

is called the outer limit of $\Phi$ as $x \rightarrow x_{0}$. If $\lim _{\sup _{x \rightarrow x_{0}}} \Phi(x) \subset$ $\Phi\left(x_{0}\right)$, then $\Phi$ is outer semicontinuous at $x_{0}$ (see [10]). It follows from the definition that $\Phi$ is outer semicontinuous if and only if $g p h \Phi$ is closed.

2.2. Some Basic Concepts and Results in Set-Valued and Variational Analysis. Given a closed set $C \subset \mathbb{R}^{n}$ and a point $\bar{x} \in \Xi$, the cone

$$
\widehat{\mathcal{N}}_{C}(\bar{x}):=\left\{\begin{array}{l|l}
x^{*} \in \mathbb{R}^{n} \mid \limsup _{\substack{\Sigma \rightarrow \bar{x} \\
x \rightarrow \bar{x}}} \frac{\left\langle x^{*}, x-\bar{x}\right\rangle}{\|x-\bar{x}\|} \leq 0
\end{array}\right\}
$$

is called the Fréchet normal cone to $C$ at $\bar{x}$. Then the limiting normal cone (also known as Mordukhovich normal cone or basic normal cone) to $C$ at $\bar{x}$ is defined by

$$
\mathcal{N}_{C}(\bar{x}):=\limsup _{x \stackrel{C}{\longrightarrow} \bar{x}} \widehat{\mathcal{N}}_{C}(x) ;
$$

see for instance [11]. It follows from the definition that the setvalued mapping $\mathcal{N}_{C}: \mathfrak{R}^{n} \rightarrow \mathcal{N}_{C}(x)$ is outer semicontinuous at $\bar{x} \in C$ and

$$
\widehat{\mathcal{N}}_{C}(\bar{x}) \subseteq \mathcal{N}_{C}(\bar{x})
$$

If inclusion (8) becomes equality, we say that $C$ is normally regular at $\bar{x}$ (or Clarke regular by [10]). According to [10, Theorem 6.9], each convex set is normally regular at all its points.

If $C=C_{1} \times C_{2}$ for closed $C_{i} \in \mathbb{R}^{n_{i}}, i=1,2$, then at any $\bar{x}=\left(\bar{x}_{1}, \bar{x}_{2}\right)$ with $\bar{x}_{i} \in C_{i}$, one has

$$
\mathcal{N}_{C}(\bar{x})=\mathcal{N}_{C_{1}}\left(\bar{x}_{1}\right) \times \mathcal{N}_{C_{2}}\left(\bar{x}_{2}\right) .
$$

For set-valued maps, the definition of coderivative was introduced by Mordukhovich in [12] based on the limiting normal cone.

Definition 1. Consider a mapping $S: \mathbb{R}^{n} \rightrightarrows \mathbb{R}^{m}$ and a point $\bar{x} \in \operatorname{dom} S$. The coderivative of $S$ at $\bar{x}$ for any $\bar{u} \in S(\bar{x})$ is the mapping $D^{*} S(\bar{x}, \bar{u}): \mathbb{R}^{m} \rightrightarrows \mathbb{R}^{n}$ defined by

$$
D^{*} S(\bar{x}, \bar{u})(y)=\left\{v:(v,-y) \in \mathcal{N}_{\mathrm{gph} S}(\bar{x}, \bar{u})\right\} .
$$


The notation $D^{*} S(\bar{x}, \bar{u})$ is simplified to $D^{*} S(\bar{x})$ when $S$ is single-valued at $\bar{x}, S(\bar{x})=\{\bar{u}\}$.

One of the tasks of variational analysis is to detect the stability of a nonlinear system when perturbations of the data occur. For this purpose, the following notations are related; see [11].

Definition 2. Consider the multifunction $F: \mathbb{R}^{m} \rightrightarrows \mathbb{R}^{n}$.

(a) (Metric regularity) we say that $F^{-1}$ is metrically regular at $\bar{x}$ for $\bar{y}$ with $(\bar{x}, \bar{y}) \in \operatorname{gph} F^{-1}$ if there are some $\tau>0$ and some neighborhoods $U$ of $\bar{x}$ and $V$ of $\bar{y}$ such that

$d(x, F(y)) \leq \tau d\left(y, F^{-1}(x)\right), \quad \forall x \in U, \quad y \in V$.

(b) (Lipschitz-like property) we say that $F$ is Lipschitzlike around $(\bar{y}, \bar{x}) \in \operatorname{gph} F$, if there exist some $\kappa>0$ and some neighborhoods $U$ of $\bar{x}$ and $V$ of $\bar{y}$ such that

$$
F\left(y^{\prime}\right) \cap U \subset F(y)+\kappa\left\|y^{\prime}-y\right\| \mathscr{B}_{n} \quad \forall y, y^{\prime} \in V .
$$

(c) (Calmness) we say that $F$ is calm at $(\bar{y}, \bar{x}) \in \operatorname{gph} F$ if there exist some $k>0$ and some neighborhoods $U$ of $\bar{x}$ and $V$ of $\bar{y}$ such that

$$
d(x, F(\bar{y})) \leq k\|y-\bar{y}\|, \quad \forall y \in V, x \in F(y) \cap U .
$$

We know from the definition that the calmness property is weaker than the Lipschitz-like property. As shown in [11], the metrical regularity of $F^{-1}$ at $\bar{x}$ for $\bar{y}$ is equivalent to the Lipschitz-like of $F$ around $(\bar{y}, \bar{x}) \in \mathrm{gph} F$, which is equivalent to the coderivative condition

$$
D^{*} F(\bar{y}, \bar{x})(0)=\{0\} .
$$

This condition is the famous Mordukhovich criterion.

2.3. Pseudometric. Let $\mathscr{B}$ denote the sigma algebra of all Borel subsets of $\Xi$ and $\mathscr{P}$ be the set of all probability measures of the measurable space $(\Xi, \mathscr{B})$ induced by $\xi$. To investigate the relationship between the solution sets of SGE (2) and (1) when the underlying probability metric $P$ varies, we need to define a metric which is closely related to the involved random functions.

Let $\mathscr{X}$ be a compact subset of $\mathbb{R}^{n}$. We start by introducing a distance function for the set $\mathscr{P}$, which is appropriate for our problem. Define the set of random functions:

$$
\mathscr{G}:=\{g(\cdot): g(\xi):=F(x, \xi), x \in \mathscr{X}\} .
$$

The distance function for any probability measures $P, Q \in \mathscr{P}$ is defined by

$$
\mathscr{D}(Q, P):=\sup _{g \in \mathscr{G}}\left\|\mathbb{E}_{P}[g(\xi)]-\mathbb{E}_{\mathrm{Q}}[g(\xi)]\right\| .
$$

This type of distance was used by Römisch (see [13, Section 2.2]) for the stability analysis of stochastic programming and was called pseudometric in that it satisfies all properties of a metric except that $\mathscr{D}(Q, P)=0$ does not necessarily imply $P=Q$ unless the set of functions $\mathscr{G}$ is sufficiently large. It is well known that $\mathscr{D}$ is nonnegative and symmetric and satisfies the triangle inequality; see [13, Section 2.1].

\section{Main Results}

In this section, we discuss existence and quantitative stability analysis of solutions to the perturbed SGE (2). To this end, we assume that $\mathscr{X} \subset \mathfrak{R}^{n}$ is a compact and convex set throughout the section and make the following assumptions.

Assumption 3. Let $\widehat{\mathscr{P}} \subset \mathscr{P}$ be a set of probability measures such that $P, Q \in \widehat{\mathscr{P}}$. The following hold:

(a) $F(x, \xi)$ is continuous at $x \in \mathfrak{R}^{n}$ for each $\xi \in \Xi$ and there exists $\kappa(\xi)$ satisfying $\sup _{P \in \widehat{\mathscr{P}}} \mathbb{E}_{P}[\kappa(\xi)]<\infty$ such that $\|F(x, \xi)\|<\kappa(\xi)$ for each $x \in \mathfrak{R}^{n}$ and $\xi \in \Xi$.

(b) $F(x, \xi)$ is continuously differentiable at $x \in \mathfrak{R}^{n}$ for each $\xi \in \Xi$ and there exists $\tau(\xi)$ satisfying $\sup _{P \in \widehat{\mathscr{P}}_{P}} \mathbb{E}_{P}[\tau(\xi)]<\infty$ such that $\left\|\mathscr{J}_{x} F(x, \xi)\right\|<\tau(\xi)$ for each $x \in \mathfrak{R}^{n}$ and $\xi \in \Xi$.

We know from [8, Theorem 7.43, 7.44] that condition (a) in Assumption 3 means that for $Q \in \widehat{\mathscr{P}}, \mathbb{E}_{\mathrm{Q}}[F(x, \xi)]$ is well defined and continuous at each $x \in \mathbb{R}^{n}$ and under condition (b) in Assumption 3, $\mathbb{E}_{\mathrm{Q}}[F(x, \xi)]$ is continuously differentiable and $\mathscr{J} \mathbb{E}_{\mathrm{Q}}[F(x, \xi)]=\mathbb{E}_{\mathrm{Q}}\left[\mathscr{J}_{x} F(x, \xi)\right]$.

3.1. Existence of a Solution to the Perturbed SGE. We now turn to discuss existence of a solution to the perturbed SGE (2). This issue has been investigated in [7] based on the results of deterministic generalized equations in Kummer [14] under the assumption of convexity of $\mathbb{E}_{P}[F(x, \xi)]+\mathscr{G}(x)$. Without this assumption, we derive the existence results based on the metric regularity.

Theorem 4. Suppose that $S(P) \neq \emptyset$ and there exist $\bar{x} \in S(P)$ such that $\bar{x} \in$ int $\mathscr{X}$. If condition (a) in Assumption 3 holds and (a) $\Psi_{P}(x)=\mathbb{E}_{P}[F(x, \xi)]+\mathscr{G}(x)$ is metrically regular at $\bar{x}$ for 0 and $(b) \Psi_{P}^{-1}(\cdot)$ is a convex-valued multifunction, then $S(Q) \neq \emptyset$ for all $Q$ close to $P$.

Proof. We know from metric regularity of $\Psi_{P}(x)=$ $\mathbb{E}_{P}[F(x, \xi)]+\mathscr{G}(x)$ at $\bar{x}$ for 0 that there exist positive constants $\gamma, \mathcal{\varepsilon}$, and $\delta$ such that

$$
d\left(x, \Psi_{P}^{-1}(q)\right) \leq \gamma d\left(q, \Psi_{P}(x)\right)
$$

for $x \in \mathscr{B}(\bar{x}, \delta) \cap \mathscr{X}$ and $q \in \mathscr{B}(0, \varepsilon)$. Since $\Psi_{P}^{-1}$ is a convexvalued multifunction, by [15, Theorem 2.2], we can define a continuous function $\widehat{x}(\cdot): \mathscr{B}(0, \varepsilon) \mapsto \mathscr{B}(\bar{x}, \delta) \cap \mathscr{X}$ such that $\widehat{x}(q) \in \Psi_{P}^{-1}(q)$ for $q \in \mathscr{B}(0, \varepsilon)$ and

$$
\|\bar{x}-\hat{x}(q)\| \leq \gamma d\left(q, \Psi_{P}(\bar{x})\right) \leq \gamma\|q\| .
$$

For each $Q$, let $\Upsilon_{Q}(x)=\mathbb{E}_{P}[F(x, \xi)]-\mathbb{E}_{Q}[F(x, \xi)]$ and $\delta^{\prime}=$ $\min \left\{\varepsilon,(1 / 2) \gamma^{-1} \delta\right\}$. Since under condition (a) in Assumption 3 ,

$$
\sup _{x \in X}\left\|\Upsilon_{Q}(x)\right\|=\mathscr{D}(Q, P) \longrightarrow 0
$$

as $Q$ is close to $P$, there exists $\delta_{1}>0$ such that

$$
\sup _{\mathrm{Q} \in U\left(P, \delta_{1}\right), x \in \mathscr{X}}\left\|\Upsilon_{\mathrm{Q}}(x)\right\| \leq \delta^{\prime} .
$$


This implies that $\Upsilon_{Q}(x) \in \mathscr{B}(0, \varepsilon)$ for $Q \in U\left(P, \delta_{1}\right)$ and $x \in X$. Therefore, by (18), we can define a function

$$
\begin{aligned}
\widehat{H}_{\mathrm{Q}}: & \mathscr{B}(\bar{x}, \delta) \cap \mathscr{X} \longrightarrow \mathscr{B}(\bar{x}, \delta) \cap \mathscr{X} \\
x & \longmapsto \widehat{x} \circ \Upsilon_{\mathrm{Q}}(x)
\end{aligned}
$$

for $Q \in U\left(P, \delta_{1}\right)$. This is a continuous mapping from the compact convex set $\mathscr{B}(\bar{x}, \delta) \cap \mathscr{X}$ to itself. By Brouwer's fixed point theorem, $\widehat{H}_{\mathrm{Q}}$ has a fixed point in $\mathscr{B}(\bar{x}, \delta)$. We assume that it is $x_{Q}$; then

$$
x_{\mathrm{Q}}=\widehat{H}_{\mathrm{Q}}\left(x_{\mathrm{Q}}\right) \in \Psi_{P}^{-1}\left(\widehat{q}_{\mathrm{Q}}\left(x_{\mathrm{Q}}\right)\right) \text {, }
$$

which means that

$$
\begin{gathered}
\mathbb{E}_{P}\left[F\left(x_{\mathrm{Q}}, \xi\right)\right]-\mathbb{E}_{\mathrm{Q}}\left[F\left(x_{\mathrm{Q}}, \xi\right)\right] \\
\in \mathbb{E}_{P}\left[F\left(x_{\mathrm{Q}}, \xi\right)\right]+\mathscr{G}(x) .
\end{gathered}
$$

Therefore,

$$
0 \in \mathbb{E}_{\mathrm{Q}}\left[F\left(x_{\mathrm{Q}}, \xi\right)\right]+\mathscr{G}(x)
$$

and hence $S(Q) \neq \emptyset$ for all $Q$ close to $P$.

Remark 5. In the case when $\mathscr{G}(x) \equiv \mathscr{K}$ in (1) with $\mathscr{K}$ being a closed convex set, we may have more simple results; that is, if $0 \in \operatorname{int}\left\{\mathbb{E}_{P}[F(\mathscr{X}, \xi)]+\mathscr{K}\right\}$, then $S(P) \neq \emptyset$ and $S(Q) \neq \emptyset$ for $Q$ close to $P$.

Corollary 6. Suppose that $\mathscr{H}(x) \equiv \mathcal{N}_{\mathscr{K}}(x)$ in (1) with $\mathscr{K}$ being a closed convex set and $\mathcal{N}_{\mathscr{K}}$ being a normal cone operator and $S(P) \neq \emptyset$. Suppose that there exist $\bar{x} \in S(P)$ such that $\bar{x} \in$ int $\mathscr{X}$ and Assumption 3 holds. If $\mathbb{E}_{P}[F(\cdot, \xi)]$ is monotone on $\mathscr{K}$ and condition

$$
\begin{aligned}
0 \in & D^{*} \mathcal{N}_{\mathscr{K}}\left(\bar{x},-\mathbb{E}_{P}[F(\bar{x}, \xi)]\right)\left(y^{*}\right) \\
& +\mathscr{J} \mathbb{E}_{P}[F(\bar{x}, \xi)]^{T}\left(y^{*}\right) \Longrightarrow y^{*}=0
\end{aligned}
$$

holds, then $S(Q) \neq \emptyset$ for $Q$ close to $P$.

Proof. We only need to verify the conditions in Theorem 4. We know from [16, Theorem 3.2] that if condition (b) in Assumption 3 holds, then under condition $(25), \Psi_{P}(x)=$ $\mathbb{E}_{P}[F(x, \xi)]+\mathcal{N}_{\mathscr{K}}(x)$ is metrically regular at $\bar{x}$ for 0 . This verifies condition (a) in Theorem 4 . Since $\mathbb{E}_{P}[F(\cdot, \xi)]$ is monotone on $\mathscr{K}$, we have

$$
\begin{aligned}
& {\left[\left(\mathbb{E}_{P}[F(x, \xi)]-q\right)-\left(\mathbb{E}_{P}[F(y, \xi)]-q\right)\right]^{T}(x-y)} \\
& \quad \geq 0
\end{aligned}
$$

for $x, y \in \mathscr{K}$, which means that $\mathbb{E}_{P}[F(\cdot, \xi)]-q$ is monotone on $\mathscr{K}$ for any $q \in \mathfrak{R}^{n}$. Then $\mathbb{E}_{P}[F(\cdot, \xi)]-q$ is monotone on $\mathscr{K}$, which, by [17, Theorem 2.3.5] and condition (a) in Assumption 3, means that $\Psi_{P}^{-1}(q)=\left\{x: q \in \mathbb{E}_{P}[F(x, \xi)]+\right.$ $\left.\mathcal{N}_{\mathscr{K}}(x)\right\}$ is a convex-valued mapping; this verifies condition (b) in Theorem 4.
Remark 7. We make some comments on the conditions in Corollary 6. (i) The condition that there exists $\bar{x} \in S(P)$ such that $\bar{x} \in \operatorname{int} \mathscr{X}$ is reasonable in that, by [17, Proposition 2.28] and its proof, $S(P)$ is nonempty if and only if there exists a closed set $\mathscr{X}$ with int $\mathscr{X} \neq \emptyset$ such that $0 \in \mathbb{E}_{P}[F(x, \xi)]+$ $\mathcal{N}_{\mathscr{K} \cap \mathscr{X}}(x)$ has a solution in int $\mathscr{X}$. In particular, $\mathscr{X}$ can be taken to a closed Euclidean ball with the fact that a solution $\bar{x}$ of $S(P)$ is the center. In this case, we know that $\bar{x}$ is also a solution of $0 \in \mathbb{E}_{P}[F(x, \xi)]+\mathcal{N}_{\mathscr{K}}(x)$. (ii) In [16, Theorem 3.2], under a calmness condition, condition (25) implies the metrical regularity of $\Psi_{P}(x)$ at $\bar{x}$ for 0 . In fact, in our case, by Definition 1, such calmness condition holds naturally by the Mordukhovich's criterion.

3.2. Stability of SGE. In this section, we undertake stability analysis of SGE (1), namely, investigating the relationship between the set of solutions to the perturbed SGE (2) and SGE (1) when probability measure $Q$ is close to $P$ under the pseudometric defined in the preceding section.

Theorem 8. Suppose that $S(P) \neq \emptyset, S(Q) \neq \emptyset$ for $Q$ close to $P$, and condition (a) in Assumption 3 holds. If $\mathscr{X} \subset \mathbb{R}^{n}$ is a convex compact set such that $S(P) \subseteq \mathscr{X}$ and $S(Q) \subseteq \mathscr{X}$ for $Q$ close to $P$, then the following assertions hold:

(i) For any small positive number $\varepsilon>0$, there exists $\delta>0$ such that

$$
S(Q) \subseteq S(P)+\varepsilon \mathscr{B}_{n} .
$$

for any $Q \in U(P, \delta)$, where $U(P, \delta)=\{Q \in \mathscr{P}$ : $\mathscr{D}(Q, P)<\delta\}$.

(ii) If $\Psi_{P}(x)=\mathbb{E}_{P}[F(x, \xi)]-\mathscr{G}(x)$ is metrically regular at any $x^{*} \in S(P)$ for 0 , then there exists $\widehat{L}>0$ such that

$$
\mathbb{D}(S(Q), S(P)) \leq \widehat{L} \mathscr{D}(Q, P)
$$

for any $Q \in U(P, \delta)$.

(iii) If $\Psi_{P}(x)=\mathbb{E}_{P}[F(x, \xi)]-\mathscr{G}(x)$ is metrically regular at any $x^{*} \in S(P)$ for 0 and $\Psi_{P}^{-1}(\cdot)$ is a convex-valued multifunction, then there exists $\widehat{L}>0$ such that

$$
\mathbb{H}(S(Q), S(P)) \leq \widehat{L} \mathscr{D}(Q, P)
$$

for any $Q \in U(P, \delta)$.

Proof. (i) Notice that $S(Q)$ is bounded for $Q$ close to $P$. The proof is directly from Theorem 3.1(ii) in [7].

Part (ii): since $\Psi_{P}$ is metrically regular at $\widehat{x}$ for 0 , there exist positive constants $\delta_{\widehat{x}}, \varepsilon_{\widehat{x}}$, and $\gamma_{\widehat{x}}$ depending on $\widehat{x}$ such that

$$
d(x, S(P)) \leq \gamma_{\bar{x}} d\left(0, \Psi_{P}(x)\right)
$$

for all $x \in S(Q) \cap \mathscr{B}\left(\widehat{x}, \delta_{\widehat{x}}\right)$. Since $x \in S(Q)$ means

$$
0 \in \mathbb{E}_{\mathrm{Q}}[F(x, \xi)]+\mathscr{G}(x)
$$


there exists $t>0$ such that for $x \in S(Q)$,

$$
\begin{gathered}
d\left(0, \Psi_{P}(x)\right) \leq \mathbb{D}\left(\mathbb{E}_{\mathrm{Q}}[F(x, \xi)]+\mathscr{G}(x)\right. \\
\left.\cap t \mathscr{B}, \mathbb{E}_{P}[F(x, \xi)]+\mathscr{G}(x) \cap t \mathscr{B}\right) \\
\leq\left\|\mathbb{E}_{\mathrm{Q}}[F(x, \xi)]-\mathbb{E}_{P}[F(x, \xi)]\right\| \leq \mathscr{D}(Q, P),
\end{gathered}
$$

which, by (30), yields

$$
d(x, S(P)) \leq \gamma_{\widehat{x}} \mathscr{D}(Q, P)
$$

for $x \in S(Q) \cap \mathscr{B}\left(\widehat{x}, \delta_{\widehat{x}}\right)$. Since $\widehat{x}$ is any choice in $S(P)$, this means that set $S(P)$ may be covered by the union of a collection of $\delta$-balls; that is,

$$
S(P) \subseteq \bigcup_{\widehat{x} \in S(Q)} \operatorname{int} \mathscr{B}\left(\widehat{x}, \delta_{\widehat{x}}\right) .
$$

Notice that $S(P)$ is a compact set, by the finite covering theorem. There exist a finite set of points $x_{1}, x_{2}, \ldots, x_{k} \in S(P)$ and a positive number $\widehat{\delta}>0$ such that

$$
S(P)+\widehat{\delta} \mathscr{B}_{n} \subseteq \bigcup_{i=1}^{k} \operatorname{int} \mathscr{B}\left(x_{i}, \delta_{x_{i}}\right)
$$

In other words, the $\widehat{\delta}$-neighborhood of $S(P)$ may be covered by a finite $\delta$-net of balls. Let $\widehat{\gamma}=\max \left\{\gamma_{x_{1}}, \ldots, \gamma_{x_{k}}\right\}$. We have from (33) that

$$
d(x, S(P)) \leq \widehat{\gamma} \mathscr{D}(Q, P)
$$

for all $x \in S(P)+\widehat{\delta} \mathscr{B}_{n}$. Through conclusion (i), we arrive at the fact that (36) holds for all $x \in S(Q)$ when $Q$ is close to $P$, which means the conclusion of (ii). that

(iii) We only need to show that there exists $L>0$ such

$$
\mathbb{D}(S(P), S(Q)) \leq L \mathscr{D}(Q, P)
$$

for $Q$ close to $P$.

Step 1. Let $\hat{x} \in S(P)$. We know from metric regularity of $\Psi_{P}$ at $\widehat{x}$ for 0 that there exist positive constants $\delta_{\widehat{x}}, \varepsilon_{\widehat{x}}$, and $\gamma_{\widehat{x}}$ depending on $\hat{x}$ such that

$$
d\left(x, \Psi_{P}^{-1}(q)\right) \leq \gamma_{\bar{x}} d\left(q, \Psi_{P}(x)\right)
$$

for all $x \in \mathscr{B}\left(\hat{x}, \delta_{\widehat{x}}\right)$ and $q \in \mathscr{B}\left(0, \varepsilon_{\widehat{x}}\right)$. Since $S(P)$ can be covered by $\left\{\mathscr{B}\left(\widehat{x}, \delta_{\widehat{x}}\right): \widehat{x} \in S(P)\right\}$, similarly to the proof in (i), there exist $\gamma>0, \delta>0$ and $\varepsilon>0$ such that

$$
d\left(x, \Psi_{P}^{-1}(q)\right) \leq \gamma d\left(q, \Psi_{P}(x)\right)
$$

for any $x \in S(P)+\delta \mathscr{B}_{n}$ and $q \in \mathscr{B}(0, \varepsilon)$.

Step 2. In particular, for fixed $\bar{x} \in S(P)$, (39) holds for any $x \in \mathscr{B}(\bar{x}, \delta)$ and $q \in \mathscr{B}(0, \varepsilon)$. Since $\Psi_{P}^{-1}$ is a convexvalued multifunction, by [15, Theorem 2.2$]$, we can define a continuous function $\tilde{x}(\cdot): \mathscr{B}(0, \varepsilon) \rightarrow \mathscr{B}(\bar{x}, \delta)$ such that $\tilde{x}(q) \in \Psi_{P}^{-1}(q)$ for $q \in \mathscr{B}(0, \varepsilon)$ and

$$
\|\bar{x}-\tilde{x}(q)\| \leq \gamma\|q\| .
$$

For each $Q$, let $\widehat{q}_{Q}(x)=\mathbb{E}_{P}[F(x, \xi)]-\mathbb{E}_{Q}[F(x, \xi)]$ and $\delta^{\prime}=$ $\min \left\{\delta, 1 / 2 \gamma^{-1} \mathcal{\varepsilon}\right\}$. Under condition (a) in Assumption 3, there exists $\delta_{1}>0$ such that

$$
\sup _{\mathrm{Q} \in U\left(P, \delta_{1}\right), x \in X}\left\|\widehat{q}_{\mathrm{Q}}(x)\right\|<\delta^{\prime},
$$

which means that $\widehat{q}_{Q}(x) \in \mathscr{B}(0, \delta)$ for $Q$ close to $P$. Let $h_{Q}(x)=\tilde{x} \circ \widehat{q}_{Q}(x)$. We know from $(40)$ that $h_{Q}(x)$ is a continuous function which maps $\mathscr{B}(\bar{x}, \delta)$ to itself uniformly for $Q$ close to $P$. By Brouwer's fixed point theorem, for each $Q$, there exist $x(Q) \in \mathscr{B}(\bar{x}, \delta)$ such that $x(Q) \in \Psi_{P}^{-1}\left(\widehat{q}_{Q}(x(Q))\right)$; that is, $x(Q) \in S(Q)$ and by $(40)$,

$$
\begin{aligned}
\|x(Q)-\bar{x}\| & =\left\|x(Q)-\tilde{x} \circ \widehat{q}_{Q}(x(Q))\right\| \\
& \leq \gamma\left\|\widehat{q}_{Q}(x(Q))\right\| \\
& \leq \gamma \sup _{Q \in U\left(P, \delta_{1}\right), x \in X}\left\|\widehat{q}_{Q}(x)\right\| \leq \gamma \mathscr{D}(Q, P)
\end{aligned}
$$

for $Q$ close to $P$, which means that

$$
d(\bar{x}, S(Q)) \leq\|x(Q)-\bar{x}\| \leq \gamma \mathscr{D}(Q, P) .
$$

Notice that $\bar{x}$ is an arbitrary choice in $S(P)$ and by (39), $\gamma$ is independent of $\bar{x}$; then we know from (43) that

$$
\mathbb{D}(S(P), S(Q)) \leq \gamma \mathscr{D}(Q, P)
$$

for $Q$ close to $P$. Combining (28) and (44), we complete the proof of (iii).

Remark 9. In [7], the error bounds described by distance from point to set are obtained to show the qualitative stability analysis of SGE (1). In Theorem 8, we establish the error bounds described by Hausdorff distance between solution sets, which extends the results of [7, Theorem 3.1].

In the case when SGE (1) has a unique solution, we need the concept of strong regularity.

Definition 10. Suppose that condition (b) in Assumption 3 holds. We say that a solution $\bar{x} \in S(P) \cap$ int $\mathscr{X}$ is strongly regular if there exist neighborhoods $\mathscr{V}$ and $\mathscr{W}$ of $0 \in \mathfrak{R}^{n}$ and $\bar{x}$, respectively, such that for every $\delta \in \mathscr{V}$, the stochastic generalized equation

$$
\delta \in \mathbb{E}_{P}[F(\bar{x}, \xi)]+\mathscr{J} \mathbb{E}_{P}[F(\bar{x}, \xi)](x-\bar{x})+\mathscr{G}(x)
$$

has a unique solution in $\mathscr{W}$ and denoted $\tilde{x}=\tilde{x}(\delta)$, and $\tilde{x}(\cdot)$ is Lipschitz continuous on $\mathscr{V}$.

In [18], consistency analysis of strong regular solution of SAA generalized equation is established. For completeness, we present a measure analogue of [18, Theorem 5.14]. 
Theorem 11. Suppose that condition (b) in Assumption 3 holds. If there exists $\bar{x}_{P} \in \operatorname{int} \mathscr{X}$ such that $S(P)=\left\{\bar{x}_{P}\right\}$ and $\bar{x}_{P}$ is strongly regular, then

(i) there exists $\varepsilon>0$ such that for every $Q \in U(P, \varepsilon), S(Q)$ contains a unique solution, denoted by $x_{\mathrm{Q}}$;

(ii) there exist constants $\varepsilon>0$ and $L>0$ such that

$$
\mathbb{D}(S(Q), S(P))=\left\|x_{Q}-x_{P}\right\| \leq \widehat{L} \mathscr{D}(Q, P)
$$

for all $Q \in U(P, \varepsilon)$.

Proof. Let $\mathscr{V}$ be a convex compact neighborhood of $\bar{x}_{P}$ and $C^{1}\left(\mathscr{V}, \Re^{n}\right)$ be a space of continuously differentiable mappings $\phi: \mathscr{V} \rightarrow \mathfrak{R}^{n}$ equipped with the norm

$$
\|\phi\|_{1, \mathscr{V}}:=\sup _{x \in \mathscr{V}}\|\phi(x)\|+\sup _{x \in \mathscr{V}}\|\mathscr{F} \phi(x)\| .
$$

Since $\bar{x}_{P}$ is a strongly regular solution of the stochastic generalized equation, there exists $\epsilon>0$ such that for any $u \in C^{1}\left(\mathscr{V}, \mathfrak{R}^{n}\right)$ satisfying $\left\|u-\mathbb{E}_{P}[F(\cdot, \xi)]\right\|_{1, \mathscr{V}} \leq \epsilon, 0 \in$ $u(x)+\mathscr{G}(x)$ has a unique solution $\widehat{x}=\widehat{x}(u) \in \mathscr{V}$ such that $\widehat{x}(\cdot)$ is Lipschitz continuous with a Lipschitz modular, denoted by $L$ with respect to the norms $\|\cdot\|_{1, \mathscr{V}}$ and

$$
\left\|\widehat{x}(u)-\widehat{x}_{P}\right\| \leq L\|u\|_{1, \mathscr{V}} .
$$

Since

$$
\left\|\mathbb{E}_{P}[F(\cdot, \xi)]-\mathbb{E}_{\mathrm{Q}}[F(\cdot, \xi)]\right\|_{1, \mathscr{V}} \longrightarrow 0
$$

as $Q$ is close to $P$, then there exists $\delta>0$ such that $S(Q)$ has a unique solution in $\mathscr{V}$ as $Q \in U(P, \delta)$. This proves (i). The conclusion of (ii) is directly from (48).

\section{Application to Stochastic Conic Programming}

Consider the following stochastic optimization problem:

$$
\begin{array}{ll}
\min _{x \in \Re^{n}} & \mathbb{E}_{P}[f(x, \xi(\omega))] \\
\text { s.t. } & \mathbb{E}_{P}[G(x, \xi(\omega))] \in \mathscr{K} \\
& x \in \mathscr{X},
\end{array}
$$

where $\mathscr{X}$ is a compact and convex subset of $\mathbb{R}^{n}, \mathscr{K}$ is a closed convex cone in $\Re^{m}, f: \mathbb{R}^{n} \times \mathbb{R}^{k} \rightarrow \mathbb{R}$ and $G: \mathbb{R}^{n} \times \mathbb{R}^{k} \rightarrow \mathbb{R}^{m}$ are continuous functions, $\xi: \Omega \rightarrow \Xi$ is vector of random variables defined on probability space $(\Omega, \mathscr{F}, P)$ with support set $\Xi \subset \mathbb{R}^{k}$, and $\mathbb{E}_{P}[\cdot]$ denotes mathematical expectation with respect to probability measure. Model (50) can be found in [2] and the linear-quadratic tracking problem is a special case of this model.

In this section, we focus on a special case when the probability measure $P$ is approximated by a sequence of empirical measures $P_{N}$ defined as

$$
P_{N}:=\frac{1}{N} \sum_{i=1}^{N} \mathbb{1}_{\xi^{k}}(\omega),
$$

where $\xi^{1}, \ldots, \xi^{N}$ is an independent and identically distributed sampling of $\xi$ and

$$
\mathbb{1}_{\xi^{k}}(\omega)= \begin{cases}1, & \text { if } \xi(\omega)=\xi^{k}(\omega), \\ 0, & \text { if } \xi(\omega) \neq \xi^{k}(\omega) .\end{cases}
$$

In this case,

$$
\begin{aligned}
& \mathbb{E}_{P_{N}}[f(x, \xi(\omega))]=\frac{1}{N} \sum_{i=1}^{N} f\left(x, \xi^{i}\right), \\
& \mathbb{E}_{P_{N}}[G(x, \xi(\omega))]=\frac{1}{N} \sum_{i=1}^{N} G\left(x, \xi^{i}\right) .
\end{aligned}
$$

By classical law of large numbers in statistics, $\mathbb{E}_{P_{N}}[f(x, \xi(\omega))]$ and $\mathbb{E}_{P_{N}}[G(x, \xi(\omega))]$ converge to $\mathbb{E}_{P}[f(x, \xi(\omega))]$ and $\mathbb{E}_{P}[G(x, \xi(\omega))]$, respectively, as $N$ tends to infinity. This kind of approximation is known in stochastic programming under various names such as sample average approximation (SAA), Monte Carlo method, and sample path optimization; see $[4,8]$ and the references therein. Consequently, by the SAA method, problem (50) can be approximated by the following problem:

$$
\begin{array}{ll}
\min _{x \in \Re^{n}} & \widehat{f}_{N}(x):=\frac{1}{N} \sum_{i=1}^{N} f\left(x, \xi^{i}\right) \\
\text { s.t. } & \widehat{G}_{N}(x):=\frac{1}{N} \sum_{i=1}^{N} G\left(x, \xi^{i}\right) \in \mathscr{K} \\
& x \in \mathscr{X} .
\end{array}
$$

Problem (54) is called the SAA problem and (50) the true problem.

If condition (b) in Assumption 3 holds for mappings $f(\cdot, \xi)$ and $G(\cdot, \xi)$, respectively, $G(\cdot, \xi)$ is convex with respect to $-\mathscr{K}$ for each $\xi$, that is, the multifunction $x \mapsto G(x, \xi)-\mathscr{K}$ is convex for each $\xi$, and the constraint qualification

$$
0 \in \operatorname{int}\left\{\mathbb{E}_{P}[G(\mathscr{X}, \xi(\omega))]-\mathscr{K}\right\}
$$

holds, then by [19, Proposition 2.104, Theorem 3.9], for any locally optimal solution of $x^{*}$, there exists $\lambda^{*} \epsilon$ $\mathcal{N}_{\mathscr{K}}\left(\mathbb{E}_{P}\left[G\left(x^{*}, \xi\right)\right]\right)$ such that $\left(x^{*}, \lambda^{*}\right)$ satisfies the following stationary condition:

$$
\begin{aligned}
0 \in & \nabla \mathbb{E}_{P}\left[f\left(x^{*}, \xi\right)\right]+\mathscr{J} \mathbb{E}_{P}\left[G\left(x^{*}, \xi\right)\right]^{T} \lambda^{*} \\
& +\mathcal{N}_{\mathscr{X}}\left(x^{*}\right),
\end{aligned}
$$

which can be rewritten as a stochastic generalized equation

$$
0 \in \mathbb{E}_{P}\left[F\left(x^{*}, \lambda^{*}, \xi\right)\right]+\mathcal{N}_{\Theta}\left(x^{*}, \lambda^{*}\right)
$$

where $F(x, \xi)=\left(\begin{array}{c}L(x, \lambda, \xi) \\ G(x, \xi)\end{array}\right)$ with $L(x, \lambda, \xi):=\nabla f(x, \xi)+$ $\mathscr{J}_{x} G(x, \xi)^{T} \lambda$ and $\Theta:=\mathscr{X} \times \mathscr{K}^{-}$, where $\mathscr{K}^{-}$is the polar cone of $\mathscr{K}$. Similarly, for fixed $N$, if $\widehat{x}_{N}$ is the locally optimal solution of problem (54), then under corresponding 
constraint qualification, there exists multiplier $\widehat{\lambda}_{N}$ such that $\left(\widehat{x}_{N}, \widehat{\lambda}_{N}\right)$ satisfies the following stationary condition:

$$
0 \in \widehat{F}_{N}\left(\widehat{x}_{N}, \widehat{\lambda}_{N}\right)+\mathcal{N}_{\Theta}\left(\widehat{x}_{N}, \widehat{\lambda}_{N}\right),
$$

where $\widehat{F}_{N}(x, \xi)=\left(\begin{array}{c}\widehat{L}_{N}(x, \lambda) \\ \widehat{G}_{N}(x)\end{array}\right)$ with $\widehat{L}_{N}(x, \lambda):=\nabla \widehat{f}_{N}(x)+$ $\mathscr{J} \widehat{G}_{N}(x)^{T} \lambda$ and $\Theta:=\mathscr{X} \times \mathscr{K}^{-}$.

Let $\Lambda_{0}=\left\{\lambda \in \mathfrak{R}^{m}: \exists x \in \mathscr{X}\right.$ s.t. $0 \in \mathbb{E}_{P}[F(x, \lambda, \xi)]+$ $\left.\mathcal{N}_{\Theta}(x, \lambda)\right\}$ and $\Lambda_{N}=\left\{\lambda \in \mathfrak{R}^{m}: \exists x \in \mathscr{X}\right.$ s.t. $0 \in \widehat{F}_{N}(x, \lambda)+$ $\left.\mathcal{N}_{\Theta}((x, \lambda))\right\}$. Next we show that all the two sets are bounded.

Lemma 12. If condition (b) in Assumption 3 holds for mappings $f(\cdot, \xi)$ and $G(\cdot, \xi)$, respectively, $G(\cdot, \xi)$ is convex with respect to $-\mathscr{K}$ for each $\xi$, and condition (55) holds, then there exist a compact set $\Lambda$ and a number $\bar{N} \in \mathbb{N}$ such that $\Lambda_{0} \cup$ $\left(\cup_{N \geq \bar{N}} \Lambda_{N}\right) \subseteq \Lambda$ almost surely.

Proof. We at first show that there exists a number $\bar{N} \in \mathbb{N}$ such that $\cup_{N \geq \bar{N}} \Lambda_{N}$ is bounded almost surely. Assume by contradiction that there exists a sequence $\left\{\lambda_{N}\right\}$ satisfying $\lambda_{N} \in \Lambda_{N}$ and $\left\|\lambda_{N}\right\| \rightarrow+\infty$ with probability one (w.p.1) as $N \rightarrow+\infty$. Then there exist a sequence $\left\{x_{N}\right\} \subseteq X$ and a number sequence $\left\{\alpha_{N}\right\} \searrow 0$ such that

$$
0 \in \nabla \widehat{f}_{N}\left(x_{N}\right)+\mathscr{L} \widehat{G}_{N}\left(x_{N}\right)^{T} \lambda_{N}+\mathscr{N}_{\mathscr{X}}\left(x_{N}\right)
$$

with $\lambda_{N} \in N_{K}\left(\widehat{G}_{N}\left(x_{N}\right)\right)$ and $\alpha_{N} \lambda_{N} \rightarrow \bar{\lambda} \neq 0$ w.p.1 as $N \rightarrow+\infty$. Under condition (b) in Assumption 3, we have by the law of large numbers that $\nabla \widehat{f}_{N}(x), \mathscr{L} \widehat{G}_{N}(x)$, and $\widehat{G}_{N}(x)$ converge to $\nabla \mathbb{E}_{P}[f(x, \xi)], \mathscr{J} \mathbb{E}_{P}[G(x, \xi)]$, and $\mathbb{E}_{P}[G(x, \xi)]$ w.p.1, respectively. Since $\mathscr{X}$ is a compact set, without loss of generality, we may assume that $x_{N} \rightarrow \bar{x}$ w.p.1 as $N$ tends to infinity. Then by the outer semicontinuity of normal cone, it holds that

$$
\begin{aligned}
\limsup _{N \rightarrow \infty} \mathcal{N}_{X}\left(x_{N}\right) \subseteq \mathcal{N}_{X}\left(x^{*}\right) \quad \text { w.p. } 1, \\
\limsup _{N \rightarrow \infty} \mathcal{N}_{\mathscr{K}}\left(\widehat{G}_{N}\left(x_{N}\right)\right) \subseteq \mathcal{N}_{\mathscr{K}}\left(\mathbb{E}_{P}\left[G\left(x^{*}, \xi\right)\right]\right)
\end{aligned}
$$

w.p.1.

Multiplying $\alpha_{N}$ by the two sides of (59) and letting $N$ tend to infinity, we obtain

$$
0 \in \mathscr{J} \mathbb{E}_{P}\left[G\left(x^{*}, \xi\right)\right]^{T} \bar{\lambda}+\mathcal{N}_{X}\left(x^{*}\right)
$$

with $\bar{\lambda} \in \mathscr{N}_{\mathscr{K}}\left(\mathbb{E}_{P}\left[G\left(x^{*}, \xi\right)\right]\right)$. Since condition (55) holds, we have from [19, Proposition 2.104] that

$$
\begin{aligned}
0 & \in \operatorname{int}\left\{\mathbb{E}_{P}\left[G\left(x^{*}, \xi\right)\right]+\mathscr{J} \mathbb{E}_{P}\left[G\left(x^{*}, \xi\right)\right]\left(\mathscr{X}-x^{*}\right)\right. \\
& -\mathscr{K}\}
\end{aligned}
$$

holds, which, by [20, Proposition 2.2], is equivalent to

$$
\begin{aligned}
0 & \in \mathcal{J} \mathbb{E}_{P}\left[G\left(x^{*}, \xi\right)\right]^{T} \lambda+\mathcal{N}_{X}\left(x^{*}\right) \\
\lambda & \in \mathcal{N}_{\mathscr{K}}\left(\mathbb{E}_{P}\left[G\left(x^{*}, \xi\right)\right]\right) \\
& \Downarrow \\
\lambda & =0 .
\end{aligned}
$$

This, by (61), means that $\bar{\lambda}=0$, which is a contradiction. Therefore there exists a number $\bar{N} \in \mathbb{N}$ such that $\cup_{N \geq \bar{N}} \Lambda_{N}$ is bounded almost surely. In the similar way, we can demonstrate that $\Lambda_{0}$ is bounded.

Let $S=\left\{(x, \lambda) \in \mathfrak{R}^{n} \times \mathfrak{R}^{m}: 0 \in \mathbb{E}_{P}[F(x, \lambda, \xi)]+N_{\Theta}(x, \lambda)\right\}$ and $\widehat{S}_{N}=\left\{(x, \lambda) \in \mathfrak{R}^{n} \times \mathfrak{R}^{m}: 0 \in \widehat{F}_{N}(x, \lambda)+N_{\Theta}(x, \lambda)\right\}$. We want to demonstrate the quantitative stability of $S$ when it is approximated by $\widehat{S}_{N}$.

Theorem 13. If condition (b) in Assumption 3 holds for mappings $f(\cdot, \xi), G(\cdot, \xi), \nabla_{x} f(\cdot, \xi), \mathscr{J}_{x} G(\cdot, \xi)$, respectively, $G(\cdot, \xi)$ is convex with respect to $-\mathscr{K}$ for each $\xi$ and condition (55) holds. If condition

$$
\begin{aligned}
& 0 \in D^{*} \mathcal{N}_{\mathscr{X}}\left(x,-\mathbb{E}_{P}[L(x, \lambda, \xi)]\right)(u) \\
&+\mathscr{J}_{x} \mathbb{E}_{P}[L(x, \lambda, \xi)](u) \\
&+\mathscr{J} \mathbb{E}_{P}[G(x, \xi)]^{T}(v) \\
& 0 \in D^{*} \mathcal{N}_{\mathscr{K}}\left(\lambda,-\mathbb{E}_{P}[G(x, \xi)]\right)(v) \\
&+\mathcal{J} \mathbb{E}_{P}[G(x, \xi)](u) \\
& \Downarrow
\end{aligned}
$$

$$
(u, v)=0
$$

holds for any $(x, \lambda) \in S$, then the following hold:

(i) There exists $\widehat{L}>0$ such that

$$
\mathbb{D}\left(\widehat{S}_{N}, S\right) \leq \widehat{L} \sup _{\phi \in \Phi} \sup _{x \in \mathscr{X}}\left\|\frac{1}{N} \sum_{i=1}^{N} \phi\left(x, \xi^{i}\right)-\mathbb{E}_{P}[\phi(x, \xi)]\right\|
$$

for $N$ is large enough, where $\Phi=\left\{G(\cdot, \xi), \nabla_{x} f(\cdot, \xi)\right.$, $\left.\mathscr{J}_{x} G(\cdot, \xi)\right\}$.

(ii) If, in addition, the matrix

$$
\left(\begin{array}{cc}
\mathscr{J}_{x} \mathbb{E}_{P}[L(x, \lambda, \xi)] & \mathscr{J}_{x} \mathbb{E}_{P}[G(x, \xi)]^{T} \\
\mathscr{J}_{x} \mathbb{E}_{P}[G(x, \xi)] & 0
\end{array}\right)
$$

is semidefinite for any $(x, \lambda) \in S$, then there exists $\widehat{L}>0$ such that

$$
\mathbb{U}\left(\widehat{S}_{N}, S\right) \leq \widehat{L} \sup _{\phi \in \Phi} \sup _{x \in \mathscr{X}}\left\|\frac{1}{N} \sum_{i=1}^{N} \phi\left(x, \xi^{i}\right)-\mathbb{E}_{P}[\phi(x, \xi)]\right\|
$$

for $N$ large enough.

Proof. It suffices to verify the conditions in Theorem 8 . By Lemma 12 , there exist a compact set $\Lambda$ and a number $\bar{N} \in \mathbb{N}$ such that $\Lambda_{0} \cup\left(\cup_{N \geq \bar{N}} \Lambda_{N}\right) \subseteq \Lambda$ almost surely, which means that $S \cup\left(\cup_{N \geq \bar{N}} \widehat{S}_{N}\right)$ contains in a compact set $\mathscr{X} \times \Lambda$ almost surely. We know from [16, Theorem 3.2] that if

$$
\begin{aligned}
0 \in & D^{*} \mathcal{N}_{\Theta}\left(x, \lambda,-\mathbb{E}_{P}[F(x, \lambda, \xi)]\right)\left(y^{*}\right) \\
& +\mathscr{J}_{x, \lambda} \mathbb{E}_{P}[F(x, \lambda, \xi)]\left(y^{*}\right) \Longrightarrow y^{*}=0,
\end{aligned}
$$


for any $(x, \lambda) \in S$, then conclusion (i) in Theorem 8 holds. Notice that by Definition 1 ,

$$
\begin{aligned}
D^{*} \mathcal{N}_{\Theta}\left(x, \lambda,-\mathbb{E}_{P}[F(x, \lambda, \xi)]\right)\left(y_{1}^{*}, y_{2}^{*}\right) \\
=D^{*} \mathcal{N}_{x}\left(x,-\mathbb{E}_{P}[L(x, \lambda, \xi)]\right)\left(y_{1}^{*}\right) \\
\quad \times D^{*} \mathcal{N}_{\mathscr{K}^{-}}\left(\lambda,-\mathbb{E}_{P}[G(x, \xi)]\right)\left(y_{2}^{*}\right)
\end{aligned}
$$

and $\mathscr{J}_{x, \lambda} \mathbb{E}_{P}[F(x, \lambda, \xi)]$ is matrix (66), which implies that (67) is equivalent to condition (64). Therefore by Theorem 8 , conclusion (i) holds.

We know from [17, Proposition 2.3.2] that if matrix (66) is semidefinite, then $\mathbb{E}_{P}[F(x, \lambda, \xi)]$ is monotone, which, by the proof of Corollary 6 , means that $\Sigma(q)=\{(x, \lambda): q \in$ $\left.\mathbb{E}_{P}[F(x, \lambda, \xi)]+N_{\Theta}(x, \lambda)\right\}$ is a convex-valued mapping; this verifies condition in (ii) of Theorem 8 . Conclusion (ii) follows from Theorem 8.

\section{Conclusion}

The existence results and quantitative stability analysis described by Hausdorff distance are established in this paper for SGE (1) when $P$ varies under some appropriate metric, which extends the results in [7]. The obtained results are then applied to a stochastic conic programming. In fact, Hausdorff distance type quantitative stability analysis obtained may be applied to more stochastic models such as stochastic mathematical program with equilibrium constraints (SMPEC) and stochastic semi-infinite programming. We let this be our further research topic.

\section{Conflicts of Interest}

The authors declare that they have no conflicts of interest.

\section{Acknowledgments}

This paper is supported by the NSFC under Projects no. 11671183 and no. 11671184 and Program for Liaoning Innovation Talents in University under Project no. LR2017049.

\section{References}

[1] S. M. Robinson, "Generalized equations and their solutions. Part I: Basic theory," Mathematical Programming Studies, vol. 10, pp. 128-141, 1979.

[2] A. J. King and R. T. Rockafellar, "Asymptotic theory for solutions in statistical estimation and stochastic programming," Mathematics of Operations Research, vol. 18, no. 1, pp. 148-162, 1993.

[3] H. Jiang and H. Xu, "Stochastic approximation approaches to the stochastic variational inequality problem," Institute of Electrical and Electronics Engineers Transactions on Automatic Control, vol. 53, no. 6, pp. 1462-1475, 2008.

[4] H. Xu, "Sample average approximation methods for a class of stochastic variational inequality problems," Asia-Pacific Journal of Operational Research, vol. 27, no. 1, pp. 103-119, 2010.
[5] H. Xu and J. J. Ye, "Approximating stationary points of stochastic mathematical programs with equilibrium constraints via sample averaging," Set-Valued and Variational Analysis, vol. 19, no. 2, pp. 283-309, 2011.

[6] S. Lu and A. Budhiraja, "Confidence regions for stochastic variational inequalities," Mathematics of Operations Research, vol. 38, no. 3, pp. 545-568, 2013.

[7] Y. Liu, W. Römisch, and H. Xu, "Quantitative stability analysis of stochastic generalized equations," SIAM Journal on Optimization, vol. 24, no. 1, pp. 467-497, 2014.

[8] A. Shapiro, D. Dentcheva, and A. Ruszczynski, Lectures on Stochastic Programming: Modeling and Theory, Society for Industrial and Applied Mathematics (SIAM), Philadelphia, PA, 2009.

[9] L.-W. Zhang, J. Zhang, and Y. Wu, "On the convergence of coderivative of SAA solution mapping for a parametric stochastic generalized equation," Set-Valued and Variational Analysis, vol. 19, no. 1, pp. 107-134, 2011.

[10] R. T. Rockafellar and R. J. B. Wets, Variational Analysis, Berlin Heidelberg, 1998.

[11] B. S. Mordukhovich, Variational Analysis and Generalized Differentiation. I: Basic Theory II : Applications, Springer, Berlin, Germany, 2006.

[12] B. S. Mordukhovich, "Metric approximations and necessary optimality conditions for general classes of extremal problems," Soviet Mathematics Doklady, vol. 22, pp. 526-530, 1980.

[13] W. Römisch, "Stability of stochastic programming problems," in Stochastic Programming. Handbooks in Operations Research and Management Science, A. Ruszczynski and A. Shapiro, Eds., vol. 10, pp. 483-554, Elsevier, Amsterdam, 2003.

[14] B. Kummer, "Generalized equations: solvability and regularity," Mathematical Programming, no. 21, pp. 199-212, 1984.

[15] A. L. Dontchev, "A local selection theorem for metrically regular mappings," Journal of Convex Analysis, vol. 11, no. 1, pp. 81-94, 2004.

[16] J. V. Outrata, Mathematical programs with equilibrium constraints: Theory and numerical methods, Springer, Vienna, 2006.

[17] F. Facchinei and J.-S. Pang, Finite-Dimensional Variational Inequalities and Complementarity Problems I-II, Springer-Verlag, New York, NY, USA, 2003.

[18] A. Shapiro, "Sensitivity analysis of parameterized variational inequalities," Mathematics of Operations Research, vol. 30, no. 1, pp. 109-126, 2005.

[19] J. F. Bonnans and A. Shapiro, Perturbation Analysis of Optimization Problems, Springer, New York, NY, USA, 2000.

[20] J. Zhang, L. Zhang, and L. Pang, "On the convergence of coderivative of SAA solution mapping for a parametric stochastic variational inequality," Set-Valued and Variational Analysis, vol. 20, no. 1, pp. 75-109, 2012. 


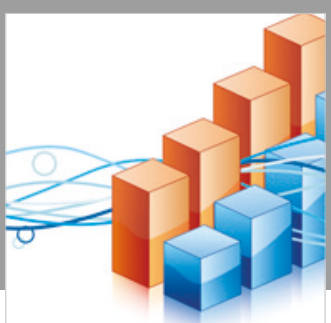

Advances in

Operations Research

\section{-n-m}
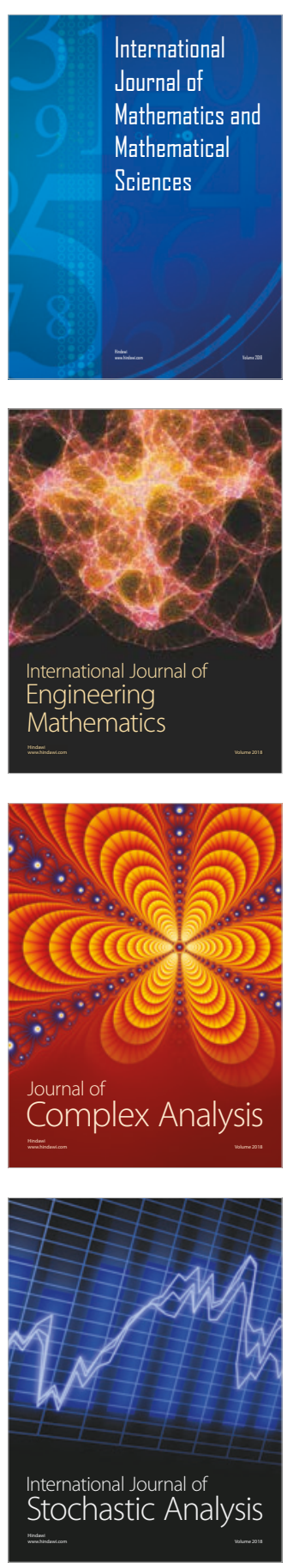
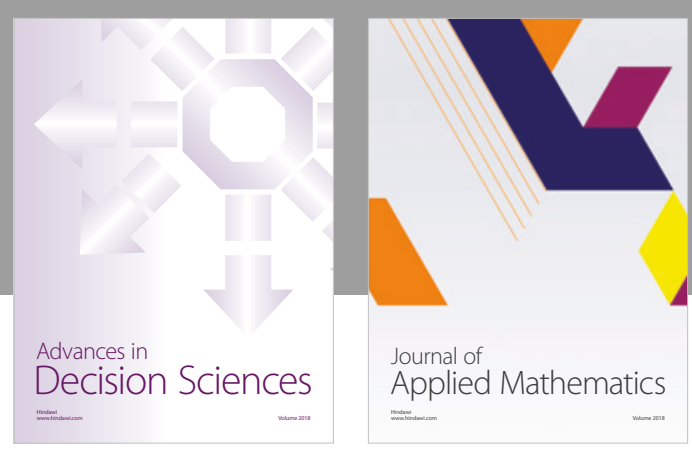

Journal of

Applied Mathematics
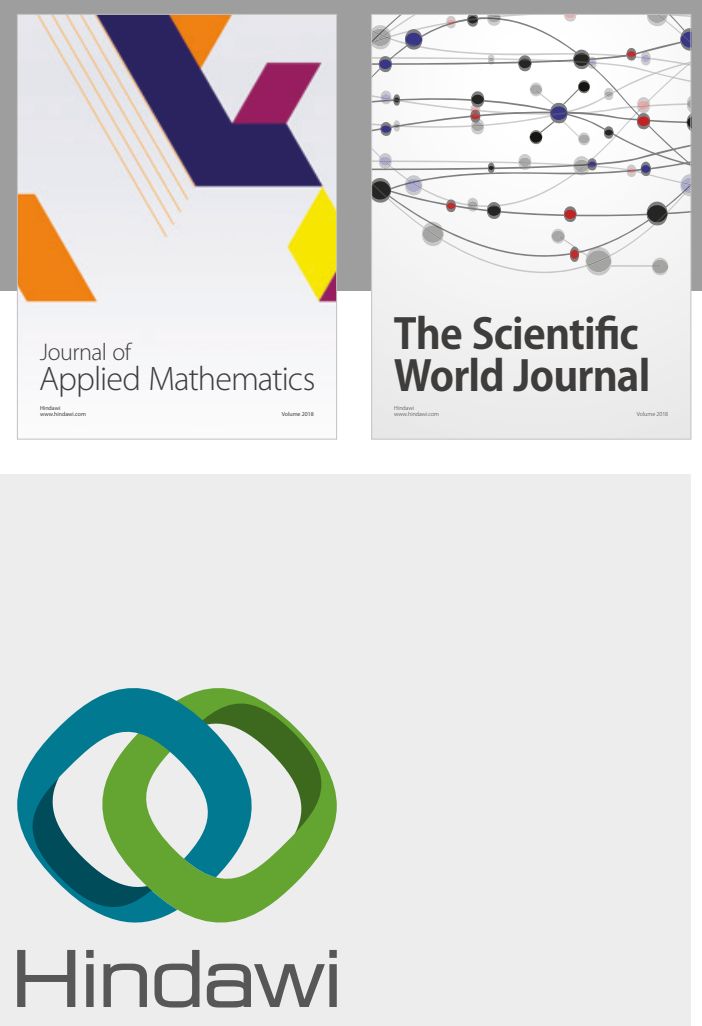

Submit your manuscripts at

www.hindawi.com

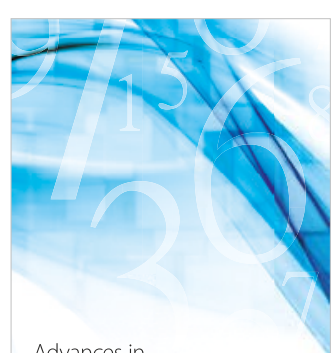

Advances in
Numerical Analysis
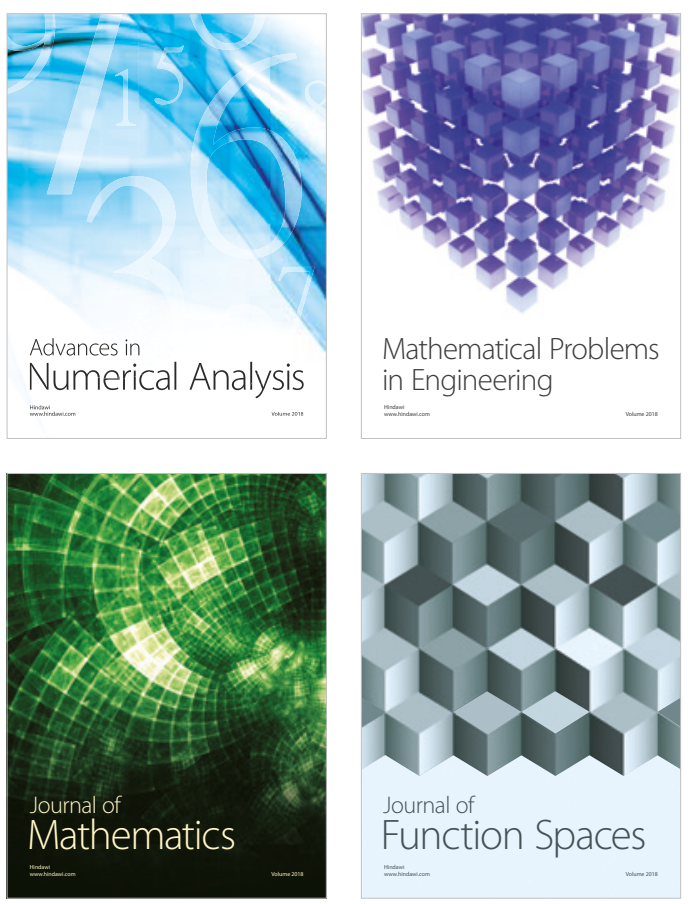

Mathematical Problems in Engineering

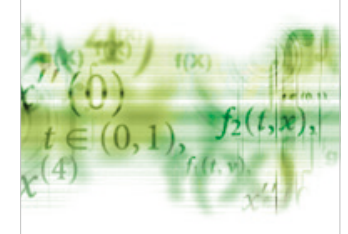

International Journal of

Differential Equations

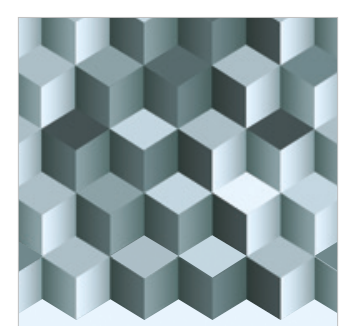

Journal of

Function Spaces

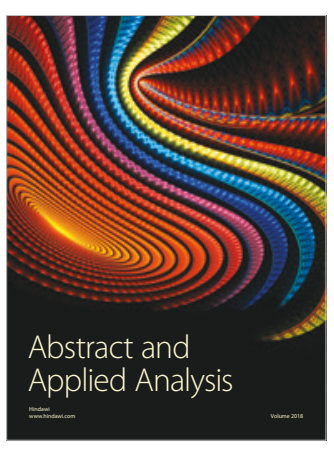

The Scientific

World Journal

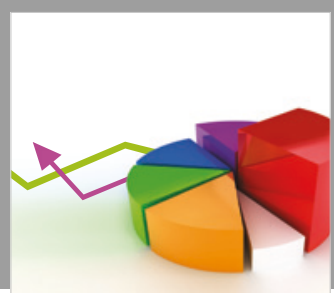

Journal of

Probability and Statistics
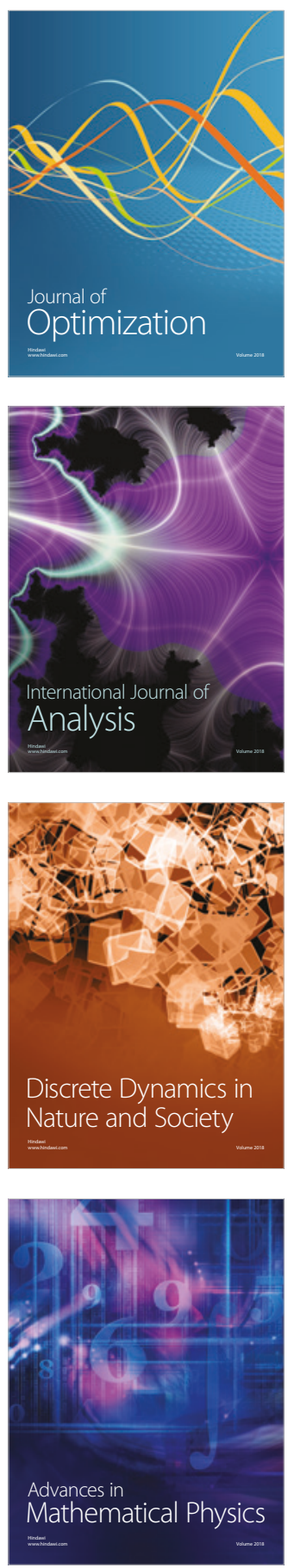